\title{
Food Intake Circadian Chronology: A Healthy Rising Science
}

\author{
Nikkhah $\mathrm{A}^{*}$ \\ Department of Animal Sciences, Faculty of Agricultural Sciences, University of Zanjan
}

${ }^{*}$ Corresponding author: Nikkhah A, Chief Highly Distinguished Professor, Department of Animal Sciences Faculty of Agricultural Sciences, University of Zanjan, Zanjan 313-45195, Iran, Fax: +98-24-35053202, Tel: +98-24-35052801, E-mail: nikkhah@znu.ac.ir

Citation: Nikkhah A (2014) Food Intake Circadian Chronology: A Healthy Rising Science. J Nutri Health Sci 1(2): 202. doi: 10.15744/2393-9060.1.202

Received Date: June 10, 2014 Accepted Date: June 25, 2014 Published Date: August 08, 2014

This editorial establishes a circadian chronological basis for food intake orchestration in modern and postmodern humans, and illuminates roadmaps for future real-world research.

Chronophysiology is an evolutionary multiscience that enables animals including humans to cope with the fluctuating environment. Timing of food intake has been discovered to orchestrate postprandial circadian rhythms of nutrient ingestion, digestion, transport, and metabolism. As such, chrono-orchestration of food intake regulates appetite and eating rate after Chrononutritional physiology is a major unnoticed healthy science that, in light of realistic modeling and understanding of voluntary feed intake in food-producing ruminant models, offers practical perceptions towards establishing health-improving feasible nutritional programs and regimens [1-5]. This is crucial considering that reliable hunger and nutrient intake predictions are indispensable to healthy and onchophobic provision of foods and nutrients to human cells. Such insightful knowledge can help formulate guidelines to prevent overnutrition and health issues namely overweight gain, obesity, and diabetes [4].

The evolutionary patterns of food searching and intake behavior have contributed to circannual and circadian patterns in endocrinology and metabolism. With the severe modifications in life style due to transition from tradition towards modernity and postmodernity, natural rhythms of appetite and nutrient intake have changed. As a result of less natural eating and overnutrition particularly fats and sugars overnutrition, it has become difficult to maximize synchronies between external environments and internal physiological states. Untimely food intake has exacerbated the modern problems by increasing risks of obesity, glucose intolerance, insulin resistance, diabetes mellitus, and resulting cardiovascular irregularities [3].

Timing of food intake as a major feasible life manager significantly affects how quickly nutrients are ingested, how extensively they are assimilated, and how proportionately the resulting substrates are distributed among different functions (i.e., deposition, oxidation, secretion, and excretion). These mechanisms are real-life scientific tools whereby the modern man will be able to optimize life quality and satisfaction, health indices, and longevity. However, circadian food intake chronology has not yet been accommodated in dietary reference intake guidelines [6].

Therefore, chronobiological management practices (e.g., circadian food intake timing and frequency) offer viable and pragmatic models to improve nutrient transformation and utilization. These strategies can well be practiced by all, particularly overweight people, diabetics, shift-workers, and athletes preparing for professional matches and games. Future research will need to explore data on nutrigenomics and immunopathology of food intake chronology. This is a path wherein medical nutrition will reveal and keep its innovative healthy disciplines on the rise.

\section{Acknowledgments}

Thanks to The Ministry of Science, Research and Technology and University of Zanjan, Iran, for supporting the author's programs of optimizing the new millennium global science education.

\section{References}

1. Nikkhah A (2013) Chronophysiology of ruminant feeding behavior and metabolism: an evolutionary review. Biol Rhythm Res 44: $197-218$.

2. Nikkhah A (2011) Bioscience of ruminant intake evolution: feeding time models. Adv Biosci Biotechnol 2: 271-4.

3. Nikkhah A (2011) Ruminant chronophysiological management: an emerging bioscience. Open Access Anim Physiol 3: 9-12.

4. Nikkhah A (2011) Science of eating time: A novel chronophysiological approach to optimize glucose-insulin dynamics and health. J Diab Mellit 2: 8-11.

5. Nikkhah A (2012) Timing of feed presentation entrains periprandial rhythms of energy metabolism indicators in once-daily fed lactating cows. Biol Rhythm Res 43: 651-61.

6. Dietary Reference Intakes (DRI) (2007) Recommended Intakes for Individuals, Food and Nutrition Board, Institute of Medicine, National Academies. National Academy of Sciences, Washington, D.C. USA. 


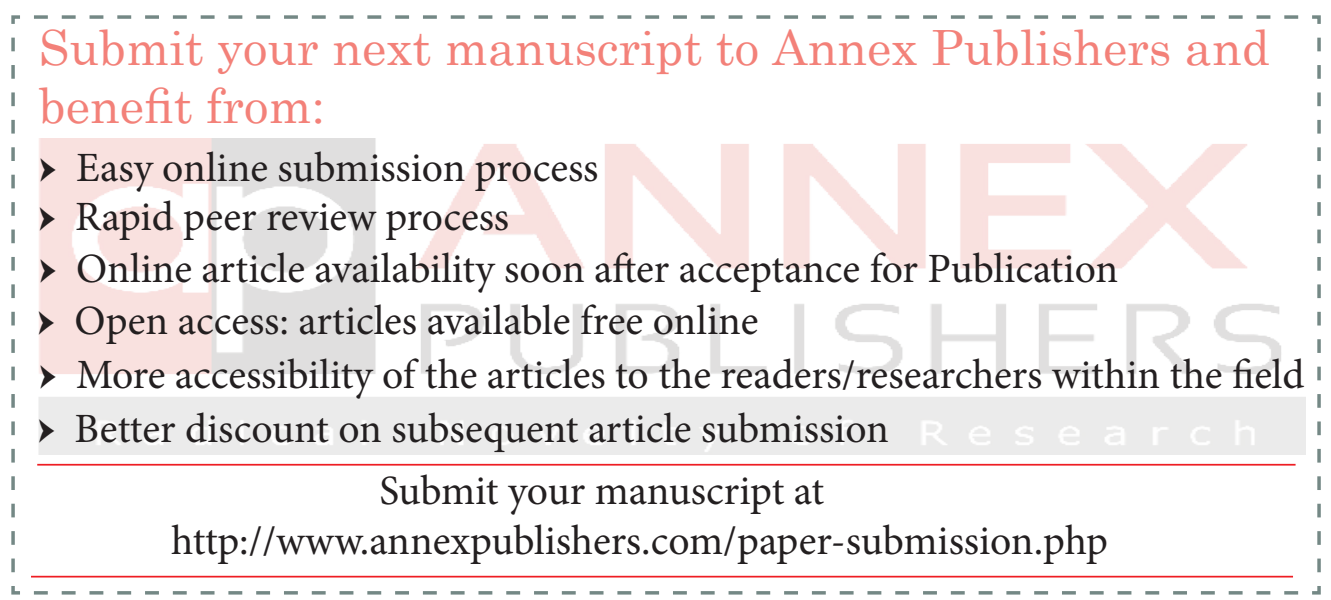

